TRANSACTIONS OF THE

AMERICAN MATHEMATICAL SOCIETY

Volume 350, Number 1, January 1998, Pages 87-100

$\mathrm{S} 0002-9947(98) 01651-1$

\title{
REALIZING HOMOLOGY BOUNDARY LINKS WITH ARBITRARY PATTERNS
}

\author{
PAUL BELLIS
}

\begin{abstract}
Homology boundary links have become an increasingly important class of links, largely due to their significance in the ongoing concordance classification of links. Tim Cochran and Jerome Levine defined an algebraic object called a pattern associated to a homology boundary link which can be used to study the deviance of a homology boundary link from being a boundary link. Since a pattern is a set of $m$ elements which normally generates the free group of rank $m$, any invariants which detect non-trivial patterns can be applied to the purely algebraic question of when such a set is a set of conjugates of a generating set for the free group. We will give a constructive geometric proof that all patterns are realized by some homology boundary link $L^{n}$ in $S^{n+2}$. We shall also prove an analogous existence theorem for calibrations of $\mathbb{E}$-links, a more general and less understood class of links tha homology boundary links.
\end{abstract}

\section{INTRODUCTION}

A link of $m$ components is a smooth, oriented, submanifold $L=\left\{K_{1}, \ldots, K_{m}\right\}$ of $S^{n+2}$ that is an ordered disjoint union of $m$ manifolds, each of which is piecewiselinearly homeomorphic to $S^{n}$. If $m=1, L$ is usually called a knot. $L$ is a boundary link if there exist $m$ disjoint Seifert surfaces, i.e. oriented submanifolds $V_{1}, \ldots, V_{m}$ of $S^{n+2}$ such that $\partial V_{i}=K_{i}$ for $i=1, \ldots, m$.

Knots and links are of interest since they repeatedly arise in the classification of manifolds. An especially significant equivalence relation on links is concordance. Two links $L_{0}$ and $L_{1}$ are concordant if there exists a smooth, oriented submanifold of $m$ components $C=\left\{C_{1}, \ldots, C_{m}\right\}$ of $S^{n+2} \times I$ such that:

(i) $C$ is piecewise-linearly homeomorphic to $L_{0} \times I$, and

(ii) $\partial C \cap\left(S^{n+2} \times\{i\}\right)=L_{i}$ for $i \in\{0,1\}$.

The classification of knot concordance groups was obtained in the mid 1960's by M. Kervaire and J. Levine [12], [14]. Among the things they prove is that the knot concordance group is trivial when $n$ is even and is an infinitely generated group when $n$ is odd. The techniques used in the concordance classification of knots have been found to extend in a compatible manner to the class of boundary links [2], [13], [19]. However, the extension of these ideas to links in general has been a much more difficult and less successful task. In the process, another class of links, called homology boundary links, has arisen. A homology boundary link of $m$ components is a link which admits $m$ disjoint generalized Seifert surfaces $\left\{Y_{1}, \ldots, Y_{m}\right\}$ such that

Received by the editors May 16, 1995 and, in revised form, October 30, 1995.

1991 Mathematics Subject Classification. Primary 57Q45, 57M07, 57M15.

(C)1998 American Mathematical Society 
$\partial Y_{i}$ is homologous to $K_{i}$ in the boundary of a tubular neighborhood of $L$. In other words, $\partial Y_{i}$ may consist of many components, each of which is an oriented longitude of some $K_{j}[20]$.

Homology boundary links have become an increasingly important class of links. In [6], [7], T. D. Cochran and K. E. Orr provided the first examples of homology boundary links which are not concordant to boundary links (cf. [9]). In [10], a scheme for classifying concordance classes of homology boundary links, analogous to that for boundary links, was presented. Further investigation has shown that sublinks of homology boundary links are in fact the "fundamental" class of links to use in the concordance classification of links [5], [16], [18]. Homology boundary links have also appeared in connection with the Alexander ideals of links [20] and in connection with the Andrews-Curtis conjecture [5].

An equivalent definition of homology boundary link is the following: Let $L$ be an $m$-component $n$-dimensional link with link group $G=\pi_{1}\left(S^{n+2}-L\right)$. Then $L$ is a homology boundary link if and only if there exists an epimorphism $\Phi$ from $G$ onto $F=\left\langle x_{1}, \ldots, x_{m}\right\rangle$, the free group on $m$ letters [20]. It is known that $L$ is a boundary link if and only if $G$ admits an epimorphism $\Phi$ (as above) with the additional condition that for some choice of meridians $\left\{\mu_{i}\right\} \subseteq G,\left\{\Phi\left(\mu_{i}\right)\right\}$ is a basis for $F$. Cochran and Levine [5] used this set $\left\{\Phi\left(\mu_{i}\right)\right\}$, which they called a pattern of a link, to study the deviance of a homology boundary link from being a boundary link. Precisely, we have:

Definition 1.1. A pattern $P=\left(r_{1}, \ldots, r_{m}\right)$ is an $m$-tuple of words in $F=$ $\left\langle x_{1}, \ldots, x_{m}\right\rangle$, a free group on $m$ letters, such that $P$ normally generates $F$. A homology boundary link $L$ admits $P$ as a pattern if there exist an epimorphism $\Phi: G=\pi_{1}\left(S^{n+2}-L\right) \rightarrow F$ and a choice of meridians $\left\{\mu_{i}\right\}$ of $L$ such that $\Phi\left(\mu_{i}\right)=r_{i}$ for $i=1, \ldots, m$.

Cochran and Levine [5] proved the following theorem algebraically:

Theorem 2.6. Given any pattern $P$ and any positive integer $n$, there exists a homology boundary link $L$ in $S^{n+2}$ admitting $P$. In particular, $L$ is a ribbon link.

In this paper, we give a constructive geometric proof of this theorem, one which provides an actual ribbon link with the desired pattern. This completes the work of Cochran and Orr on classifying homology boundary links with specified pattern and Seifert form [8]. Further, with a slight modification of the proof, we prove an analogous existence theorem for a more general class of links, namely $\mathbb{E}$-links, which are defined and discussed in $\S 3$. The class of $\mathbb{E}$-links is essentially the class of links which are sublinks of homology boundary links. Thus, according to [5], [16], [18] this is an important class of links.

Theorem 3.4. Given any finitely generated $\mathbb{E}$-group $E$, calibration $\left(E,\left\{y_{1}, \ldots\right.\right.$, $\left.\left.y_{m}\right\}\right)$, and positive integer $n$, there exists an $\mathbb{E}$-link $L$ in $S^{n+2}$ admitting the $E$ calibration $\left(E,\left\{y_{1}, \ldots, y_{m}\right\}\right)$. In particular, $L$ is a ribbon link.

As we will see, an $\mathbb{E}$-calibration is the analog to $\mathbb{E}$-links of a pattern.

\section{FUSIONS OF LINKS}

Given a link $L^{n}$ in $S^{n+2}$ and an $\operatorname{arc} b$ connecting two different components of $L$, i.e. $b$ is smoothly embedded in $S^{n+2}$ and intersects $L$ only at its endpoints (orthogonally), choose a normal vector field $\nu$ along $b$ which is normal to $L$ at both 


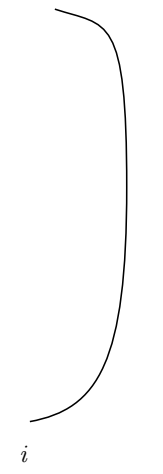

$L$

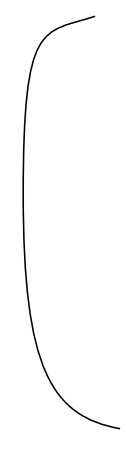

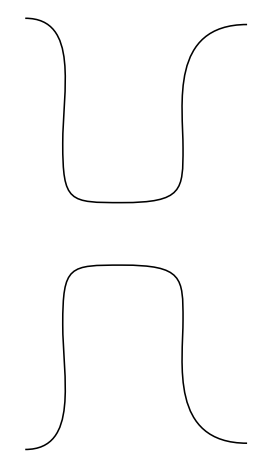

$(L)$

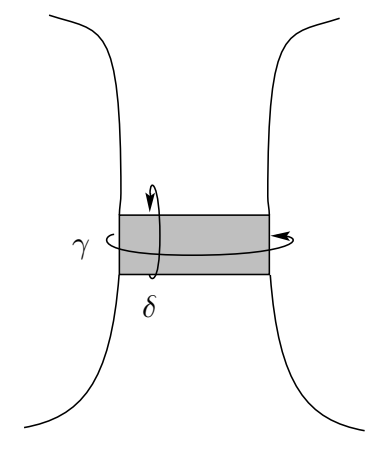

$(L)$

FiguRe 2.1

endpoints of $b$. With the proper orientation of $b$, one can perform the connected sum of the two components of $L$ along $b$ (just use the orthogonal complement of $\nu$ in a tubular neighborhood of $b$ as the connecting tube). The resulting link $F(L)$ is a link with one less component than $L$ and is called the fusion of $L$ along the band $B=\{\nu \cup b\}$. One can perform more than one fusion to a link along a collection of bands $\left\{B_{i}\right\}$, thus obtaining a sequence of fusions $F_{1}(L), \ldots, F_{k}(L) . F(L)=F_{k}(L)$ is called the fusion of a link along the bands $\left\{B_{i}\right\}$. Of particular interest is the situation when $L$ is a boundary link, and one performs a sequence of fusions of $L$ along a collection of bands $\left\{B_{i}\right\}$, resulting in the fusion of a boundary link along the bands $\left\{B_{i}\right\}$ (cf. [3], [5]). Fusions of boundary links are of interest here because one can obtain useful information relating the link groups of $L$ and $F(L)$.

Let $L$ be a link in $S^{3}, F(L)$ its fusion along a band $B$, and $B(L)$ the corresponding "band link", i.e. $B(L)=L \cup B$ as shown in Figure 2.1. There is no direct way to relate $\pi_{1}\left(S^{3}-L\right)$ and $\pi_{1}\left(S^{3}-F(L)\right)$, the link groups of $L$ and $F(L)$, respectively. However, $L$ and $F(L)$ both are subsets of $B(L)$. Hence, there exist inclusion maps of their complements $\phi: S^{3}-B(L) \rightarrow S^{3}-L$ and $\psi: S^{3}-B(L) \rightarrow S^{3}-F(L)$ and corresponding induced maps $\phi_{*}: \pi_{1}\left(S^{3}-B(L)\right) \rightarrow \pi_{1}\left(S^{3}-L\right)$ and $\psi_{*}$ : $\pi_{1}\left(S^{3}-B(L)\right) \rightarrow \pi_{1}\left(S^{3}-F(L)\right)$. The curves $\gamma$ and $\delta$ are of particular interest here. Note that $\delta \in \operatorname{ker}\left(\phi_{*}\right)$ and $\gamma \in \operatorname{ker}\left(\psi_{*}\right)$. Observe that $S^{3}-L=S^{3}-B(L) \cup 2$ handle and $S^{3}-F(L)=S^{3}-B(L) \cup 2$-handle, so the link groups of $L$ and $F(L)$ can be related as quotient groups of the "link" group $\pi_{1}\left(S^{3}-B(L)\right)$.

Decompose $S^{3}$ as the union of two 3 -balls $D_{+}^{3}$ and $D_{-}^{3}$ in such a way that $D_{-}^{3}$ is a small 3-ball containing the bands $\left\{B_{i}\right\}$. Think of $D_{+}^{3}$ and $D_{-}^{3}$ as the "outside" and "inside" of $S^{2}=D_{+}^{3} \cap D_{-}^{3}=\partial D_{+}^{3}=\partial D_{-}^{3}$. Note that for every fusion band $B_{i}$, there exist curves $\gamma_{i}$ and $\delta_{i}$ as described in Figure 2.1. Set:

(i) $U=D_{+}^{3}-L$,

(ii) $V_{1}=D_{-}^{3}-L$, and

(iii) $V_{2}=D_{-}^{3}-F(L)$.

Applying Seifert-Van Kampen twice, one obtains the following two isomorphisms:

$$
\begin{aligned}
& \Theta_{L}: \pi_{1}\left(S^{3}-L\right) \rightarrow \pi_{1}(U) /\left\langle\left\{\delta_{i}\right\}\right\rangle, \\
& \Theta_{F(L)}: \pi_{1}\left(S^{3}-F(L)\right) \rightarrow \pi_{1}(U) /\left\langle\left\{\gamma_{i}\right\}\right\rangle .
\end{aligned}
$$


Define an epimorphism $K: \pi_{1}(U) /\left\langle\left\{\gamma_{i}\right\}\right\rangle \rightarrow \pi_{1}(U) /\left\langle\left\{\gamma_{i}, \delta_{i}\right\}\right\rangle$ whose kernel is $\left\langle\left\{\delta_{i}\right\}\right\rangle$. Then

$$
\Psi=\Theta_{L}^{-1} \circ K \circ \Theta_{F(L)}: \pi_{1}\left(S^{3}-F(L)\right) \rightarrow \pi_{1}\left(S^{3}-L\right) /\left\langle\left\{\gamma_{i}\right\}\right\rangle
$$

is an epimorphism from the link group of $F(L)$ onto a quotient group of the link group of $L$. Thus, we have proved the following proposition.

Proposition 2.2. Let $F(L)$ be a fusion of a boundary link along fusion bands $\left\{B_{1}, \ldots, B_{m}\right\}$. Then there exists an epimorphism

$$
\Psi: \pi_{1}\left(S^{3} 3-F(L)\right) \rightarrow \pi_{1}\left(S^{3}-L\right) /\left\langle\left\{\gamma_{i}\right\}\right\rangle .
$$

Remarks 2.3. (1) The proof of Proposition 2.2 shows that the epimorphism $\Psi$ is defined independently of the order in which the bands are fused onto $L$.

(2) One can easily generalize Proposition 2.2 to links $L^{n}$ of higher dimensions. The proof can be altered in the obvious way so that $\gamma_{i}$ is a circle and $\delta_{i}$ is an $n$-sphere. Thus, $\Psi$ will be an isomorphism since its kernel is $\left\langle\delta_{i}\right\rangle$ (which is trivial in $\pi_{1}$ ).

(3) We can further analyze the curves $\gamma_{i}$ as follows. If the fusion band $B_{i}$ fuses together components $K_{j}$ and $K_{k}$ of $L$ and if $x_{i j}$ and $x_{i k}$ are meridional elements of the link group of $L$ for $K_{j}$ and $K_{k}$, respectively, then $\gamma_{i}=\bar{x}_{i j} \eta_{i} x_{i k} \bar{\eta}_{i}$ for $\eta_{i} \in$ $\pi_{1}\left(S^{3}-L\right)$ and where $\bar{x}_{i}=x_{i}^{-1}$ (see Figure 2.4).

This leads to the following useful corollary (cf. [3]).

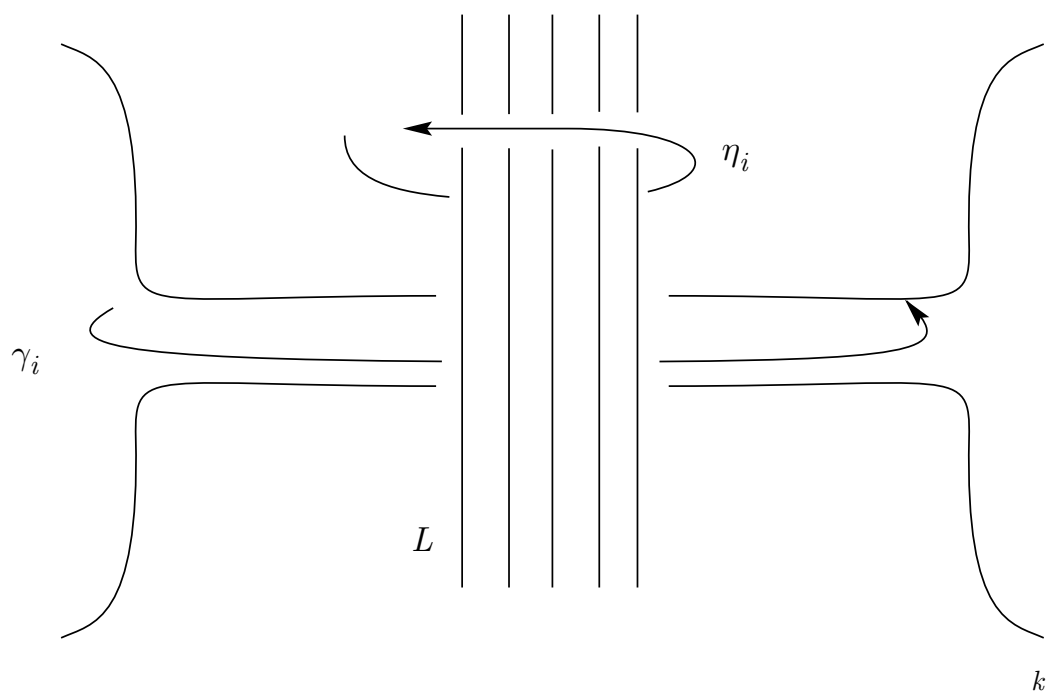

FiguRE 2.4

Corollary 2.5. Let $L=\left\{K_{1}, \ldots, K_{m}\right\}$ be an m-component trivial link, i.e. $\pi_{1}\left(S^{3}-L\right)=\left\langle x_{1}, \ldots, x_{m}\right\rangle$ where $x_{j}$ is a meridional generator of $K_{j}$. Let $F(L)$ be a fusion of $L$ along fusion bands $\left\{B_{1}, \ldots, B_{k}\right\}$, where $B_{i}$ fuses together components $K_{j}$ and $K_{k}$. Then there exists an epimorphism

$$
\Psi: \pi_{1}\left(S^{3}-F(L)\right) \rightarrow\left\langle x_{1}, \ldots, x_{m} \mid x_{i j}=\eta_{i} x_{i k} \bar{\eta}_{i}\right\rangle
$$

where $\eta_{i} \in \pi_{1}\left(S^{3}-L\right)$. 
We are now ready to present a constructive geometric proof of Cochran and Levine's theorem (cf. [9]).

Theorem 2.6. Given any pattern $P$ and any positive integer $n$, there exists a homology boundary link $L$ in $S^{n+2}$ admitting $P$. In particular, $L$ is a ribbon link.

Proof. Let $F=\left\langle x_{1}, \ldots, x_{m}\right\rangle$ be a free group and suppose $P=\left(r_{1}, \ldots, r_{m}\right)$ is a pattern in $F$. Since the map $\operatorname{Aut}(F) \rightarrow \operatorname{Aut}(F /[F, F])$ is onto, assume without loss of generality that $x_{i}=r_{i} \bmod [F, F]$ for all $i$. Since $P$ is a pattern, the elements of $P$ normally generate $F$, so each generator $x_{i}$ may be expressed as a word $w_{i}$ such that

$$
w_{i}=\prod_{j=1}^{\lambda_{i}} \eta_{i j} r_{\nu(i, j)}^{\varepsilon_{i j}} \bar{\eta}_{i j}
$$

where $r_{\nu(i, j)} \in\left\{r_{1}, \ldots, r_{m}\right\}, \eta_{i j}$ is a word $\prod_{k=1}^{\lambda_{i j}} x_{\sigma(i, j, k)}^{\varepsilon_{i j k}}$ in $\left\{x_{i}\right\}$, and $\varepsilon_{i j}, \varepsilon_{i j k} \in$ $\{ \pm 1\}$. By representing each element of the given basis of $F$ as a product of conjugates of pattern elements, we have achieved a decomposition which will appear in the construction upon application of Corollary 2.5.

Begin the construction by drawing $m$ circles one below another, as shown in Figure 2.7; call these circles $\beta_{i}$. Next to $\beta_{i}$, draw $\lambda_{i}$ concentric circles; call these circles $\alpha_{i j}$ for $1 \leq i \leq m, 1 \leq j \leq \lambda_{i j}$. Orient the $\beta_{i}$ 's all clockwise and orient $\alpha_{i j}$ clockwise or counterclockwise depending on whether the exponent $\varepsilon_{i j}$ of $r_{\nu(i, j)}$ in $w_{i}$ is +1 or -1 . Call the result $L_{0}$.

Note that $G_{0}=\pi_{1}\left(S^{3}-L_{0}\right)$ is a free group on $m+\sum_{i=1}^{m} \lambda_{i}$ letters. Let $b_{i}$ and $a_{i j}$ be simple meridional generators in $G_{0}$ for $\beta_{i}$ and $\alpha_{i j}$, respectively, oriented using the right-hand rule. Then $G_{0} \approx\left\langle b_{i}, a_{i j}\right\rangle$. Now we will add fusion bands $B_{i j}$ to $L_{0}$ to obtain the desired link $L$. Fuse a band $B_{i j}$ to $L_{0}$ starting at $\alpha_{i j}$ and connecting to $\beta_{\nu(i, j)}$. Pass the band $B_{i j}$ through the center of the $\sigma(i, j, k)$ th set of concentric circles for every $x_{\sigma(i, j, k)}$ in $\eta_{i j}$. $B_{i j}$ should pass top to bottom if the exponent $\varepsilon_{i j k}$ of $x_{\sigma(i, j, k)}$ is +1 and bottom to top if the exponent is -1 . If the circle $\alpha_{i j}$ is oriented clockwise, $B_{i j}$ must be given a half-twist before it is attached to $\beta_{j}$ to preserve orientation; if $\alpha_{i j}$ is oriented counterclockwise, no twists are necessary. Fuse such a band $B_{i j}$ to $L_{0}$ for $1 \leq i \leq m$ and $1 \leq j \leq \lambda_{i}$ in the manner described above to obtain the desired link $L$; i.e. $L$ will be the fusion of $L_{0}$ along the bands $\left\{B_{i j}\right\}$.

To show that $L$ admits $P$ as a pattern, we must exhibit an epimorphism $\Phi$ : $\pi_{1}\left(S^{3}-L\right) \rightarrow F$ such that for some choice of meridians $\left\{\mu_{i}\right\}$ of $L, \Phi\left(\mu_{i}\right)=r_{i}$ for $i=1, \ldots, m$ ( $L_{0}$ has $m+\sum_{i=1}^{m} \lambda_{i}$ components and $\sum_{i=1}^{m} \lambda_{i}$ bands were fused to $L_{0}$, so $L$ is an $m$-component link). From the construction, one observes that each component $\beta_{i}$ of $L_{0}$ lies in a different component of $L$ (so we can consider $L$ similarly ordered). Choose a set of meridians $\left\{\mu_{i}\right\}$ of $L$ such that each $\mu_{i}$ is a simple meridian of the $i$ th component of $L$ equivalent to $b_{i}$. The curve $\gamma_{i j}$ corresponding to the band $B_{i j}$, by construction, is expressed as:

$$
a_{i j}=\prod_{k=1}^{\lambda_{i j}}\left[\left(\prod_{t=1}^{\lambda_{i j k}} a_{\sigma(i, j, k) t}^{\varepsilon_{\sigma(i, j, k) t}}\right)\right]^{\varepsilon_{i j k}} b_{\nu(i, j)} \overline{\prod_{k=1}^{\lambda_{i j}}\left[\left(\prod_{t=1}^{\lambda_{i j k}} a_{\sigma(i, j, k) t}^{\varepsilon_{\sigma(i, j, k) t}}\right)\right]^{\varepsilon_{i j k}}}
$$

in $\pi_{1}\left(S^{3}-L_{0}\right)$. By Proposition 2.2, there exists an epimorphism

$$
\Psi: \pi_{1}\left(S^{3}-L\right) \rightarrow \pi_{1}\left(S^{3}-L_{0}\right) /\left\langle\gamma_{i j}\right\rangle,
$$



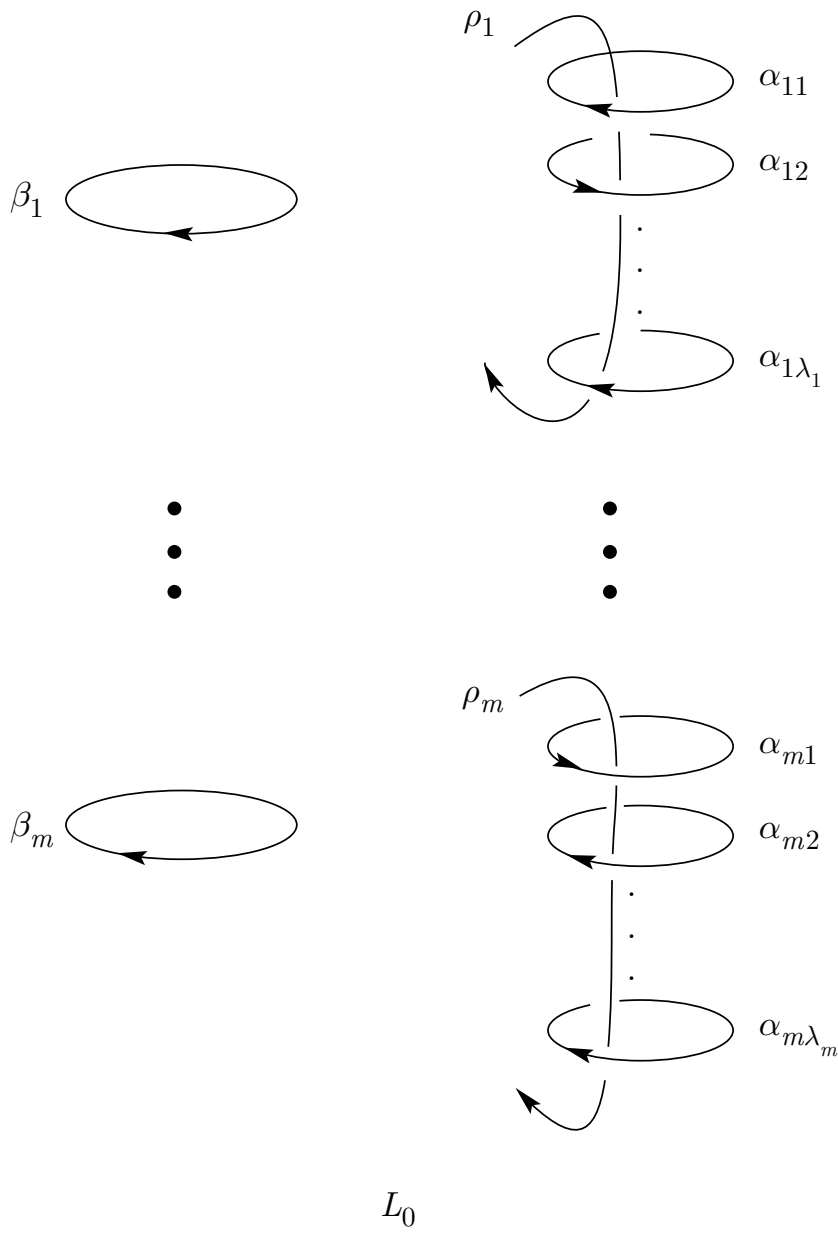

Figure 2.7

where by Corollary 2.5

$$
\begin{aligned}
& \pi_{1}\left(S^{3}-L_{0}\right) /\left\langle\gamma_{i j}\right\rangle \\
& \quad=\left\langle b_{i}, a_{i j} \mid a_{i j}=\prod_{k=1}^{\lambda_{i j}}\left[\left(\prod_{t=1}^{\lambda_{i j k}} a_{\sigma(i, j, k) t}^{\varepsilon_{\sigma(i, j, k) t}}\right)\right]^{\varepsilon_{i j k}} b_{\nu(i, j)} \prod_{k=1}^{\lambda_{i j}\left[\left(\prod_{t=1}^{\lambda_{i j k}} a_{\sigma(i, j, k) t}^{\varepsilon_{\sigma(i, k) t}}\right)\right]^{\varepsilon_{i j k}}}\right\rangle .
\end{aligned}
$$

Using Tietze transformations on the group $\pi_{1}\left(S^{3}-L_{0}\right) /\left\langle\gamma_{i j}\right\rangle$, add generators $A_{i}$ and relations $A_{i}=\prod_{j=1}^{\lambda_{i}} a_{i j}^{\varepsilon_{i j}}$. Then recall Figure 2.7 and observe that the curve $\rho_{i}$ is represented in $\pi_{1}\left(S^{3}-L_{0}\right) /\left\langle\gamma_{i j}\right\rangle$ by $\prod_{j=1}^{\lambda_{i}} a_{i j}^{\varepsilon_{i j}}$. So we have obtained the following presentation:

$$
\pi_{1}\left(S^{3}-L_{0}\right) /\left\langle\gamma_{i j}\right\rangle=\left\langle b_{i}, a_{i j}, A_{i} \mid a_{i j}=\prod_{k=1}^{\lambda_{i j}} A_{\sigma(i, j, k)}^{\varepsilon_{i j k}} b_{\nu(i, j)} \overline{\prod_{k=1}^{\lambda_{i j}} A_{\sigma(i, j, k)}^{\varepsilon_{i j k}}}, A_{i}=\prod_{j=1}^{\lambda_{i}} a_{i j}^{\varepsilon_{i j}}\right\rangle .
$$


Define a map $\Psi_{0}: \pi_{1}\left(S^{3}-L_{0}\right) /\left\langle\gamma_{i j}\right\rangle \rightarrow F$ where

$$
b_{i} \rightarrow r_{i}, \quad a_{i j} \rightarrow \eta_{i j} r_{\nu(i, j)} \bar{\eta}_{i j}, \quad \text { and } \quad A_{i} \rightarrow x_{i} .
$$

$\Psi_{0}$ is well defined since the image of relations

$$
a_{i j}=\prod_{k=1}^{\lambda_{i j}} A_{\sigma(i, j, k)}^{\varepsilon_{i j k}} b_{\nu(i, j)} \overline{\prod_{k=1}^{\lambda_{i j}} A_{\sigma(i, j, k)}^{\varepsilon_{i j k}}}
$$

are trivial in $F$, and the relations $A_{i}=\prod_{j=1}^{\lambda_{i}} a_{i j}^{\varepsilon_{i j}}$ become $x_{i}=w_{i} . \Psi_{0}$ is onto since $\left\{\Psi_{0}\left(A_{i}\right)\right\}$ is a basis for $F$. Define $\Phi: \pi_{1}\left(S^{3}-L\right) \rightarrow F$ by $\Phi=\Psi_{0} \circ \Psi$. One checks that $\Phi\left(\mu_{i}\right)=\Psi_{0}\left(b_{i}\right)=r_{i}$. Thus $L$ is a homology boundary link admitting $P$ as a pattern. One further observes that $L$ is a ribbon link by construction. $\diamond$

Remarks 2.8. (1) The link $L$ obtained in the proof is not unique. The proof only requires that fusion bands be added which do not intersect one another or the link $L_{0}$ (except where they are properly attached). The fusion band may be twisted and knotted in any fashion as long as these necessary conditions are maintained!

(2) The proof is the same for the construction of higher dimensional links (cf. Proposition 2.2, Remarks 2.3).

We close this section with two examples. The first is a relatively simple one, which provides a homology boundary link which is not concordant to a boundary link. The second example uses a pattern which is only slightly more complicated than the first example. However, the resulting link is much more complicated!

Example 2.9. Consider the free group $F=\left\langle x_{1}, x_{2}\right\rangle$ and pattern $P_{1}=\left(r_{1}, r_{2}\right)=$ $\left(x_{1}\left[x_{1}, x_{2}\right], x_{2}\right)$. Cochran and Orr constructed a link admitting this pattern which was the first (and still simplest known) example of a homology boundary link which is not concordant to a boundary link [6], [7]. Since $P_{1}$ is a pattern, $P_{1}$ normally generates $F$, so $x_{i}$ is expressible as a word $w_{i}$ where

$$
w_{i}=\prod_{j=1}^{\lambda_{i}} \eta_{i j} r_{\nu(i, j)}^{\varepsilon_{i j}} \bar{\eta}_{i j} .
$$

For $P_{1}$, we have

$$
\begin{aligned}
& \lambda_{1}=3, \quad \lambda_{2}=1, \\
& r_{\nu(1,1)}=r_{1}, \quad r_{\nu(1,2)}=r_{\nu(2,1)}=r_{2}, \quad r_{\nu(1,3)}=\bar{r}_{2}, \\
& \eta_{11}=\eta_{12}=\bar{x}_{1}, \quad \eta_{13}=\eta_{21}=1 .
\end{aligned}
$$

Applying the construction in Theorem 2.6, one obtains a homology boundary link $L_{1}$, pictured in Figure 2.10. One may also observe that $L_{1}$ is equivalent to the initial link used by Cochran and Orr, shown in Figure 2.11. 


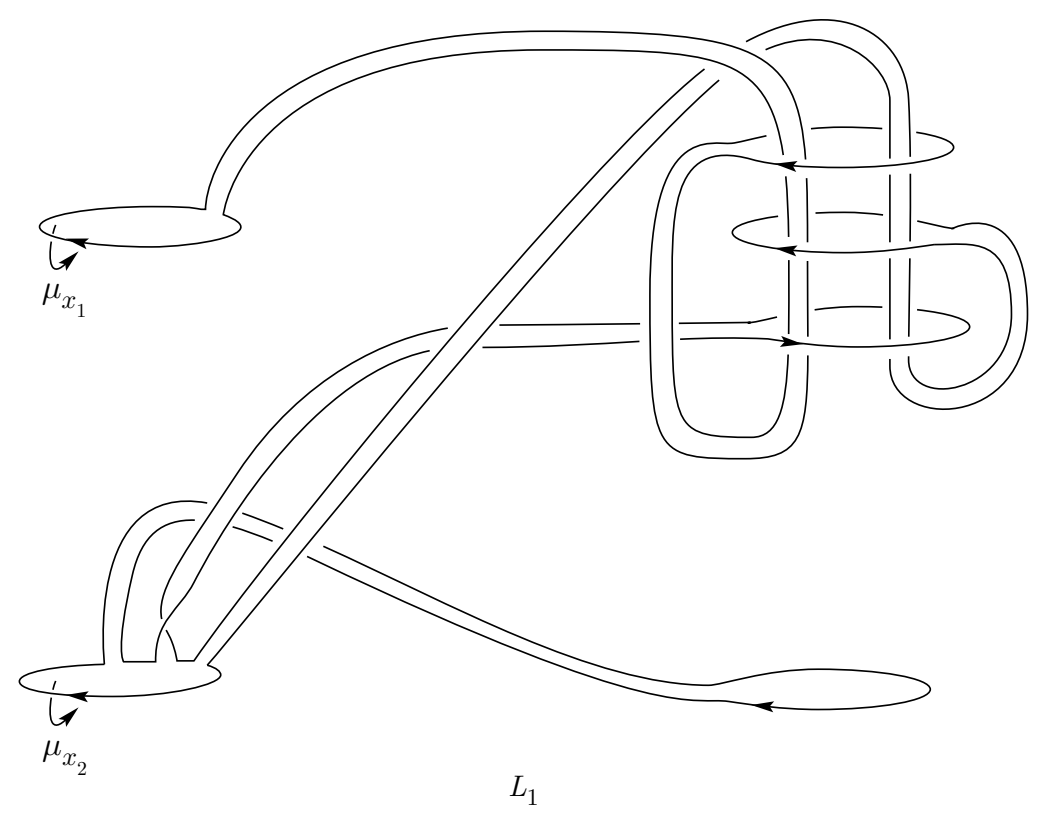

FiguRe 2.10

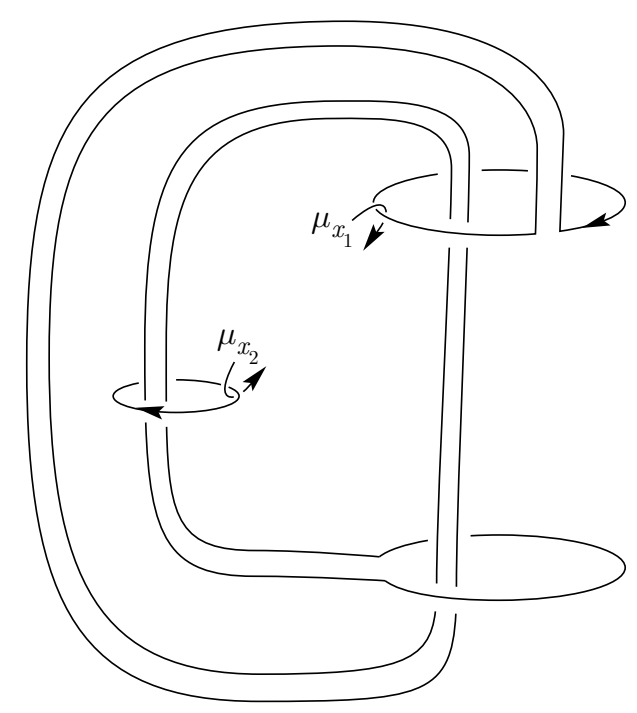

FigURE 2.11

Example 2.12. Consider the free group $F=\left\langle x_{1}, x_{2}\right\rangle$ and pattern $P_{2}=\left(r_{1}, r_{2}\right)=$ $\left(\left[x_{2}, \bar{x}_{1}\right] x_{1},\left[x_{1}, \bar{x}_{2}\right] x_{2}\right)$. Note that no element of $P_{2}$ is conjugate to any generator of $F$, so any homology boundary link admitting $P$ cannot be a boundary link [5]. Nor can $L$ be the strong fusion of a boundary link [10]. Since $P_{2}$ is a pattern, $P_{2}$ 


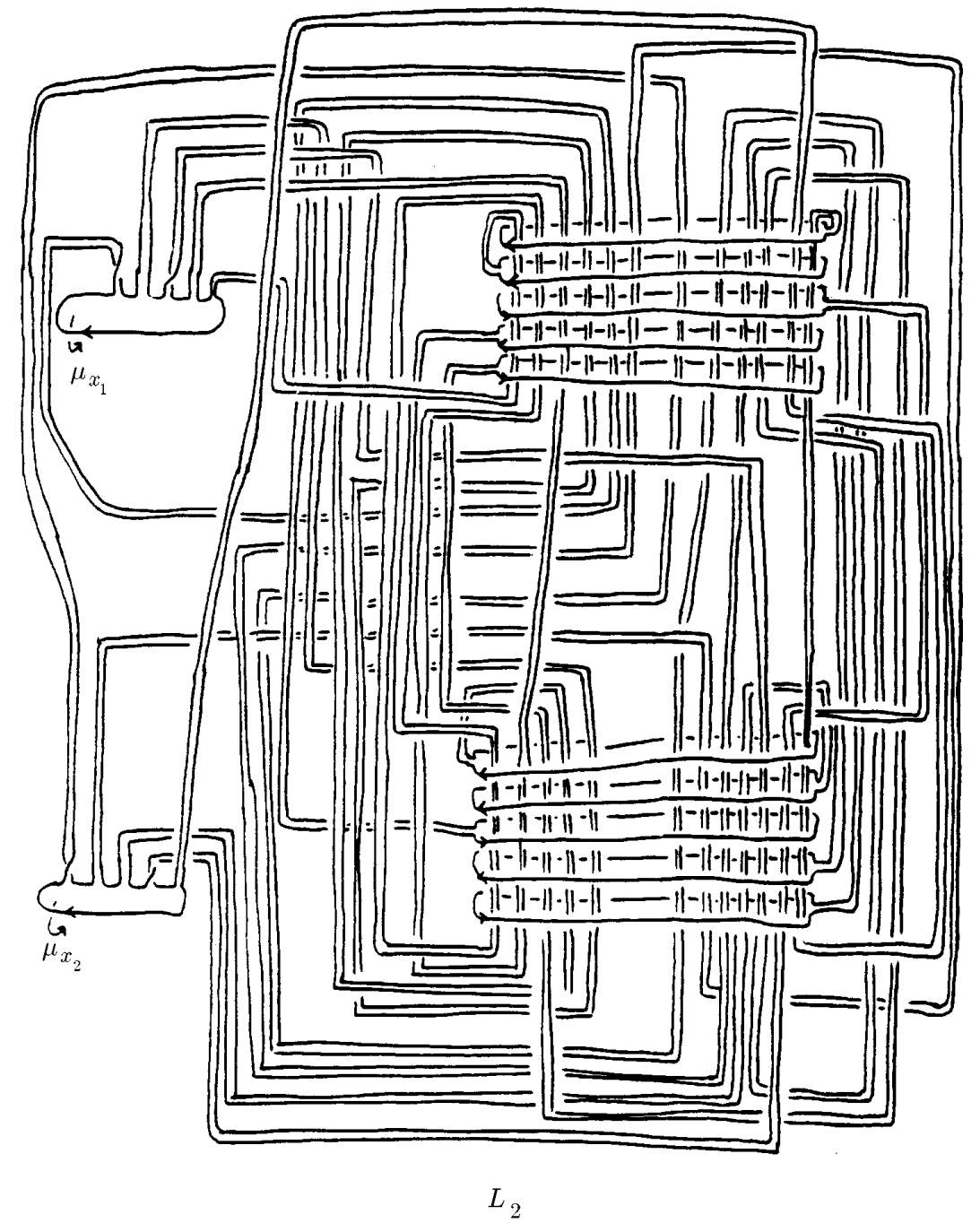

FiguRe 2.13

normally generates $F$, so $x_{i}$ is expressible as a word $w_{i}$ where

$$
w_{i}=\prod_{j=1}^{\lambda_{i}} \eta_{i j} r_{\nu(i, j)}^{\varepsilon_{i j}} \bar{\eta}_{i j} .
$$

For the pattern $P_{2}$ given above we have the following:

$$
\begin{aligned}
& \lambda_{1}=\lambda_{2}=5, \\
& r_{\nu(1,2)}=r_{\nu(1,4)}=r_{\nu(2,1)}=r_{1}, \quad r_{\nu(1,5)}=r_{\nu(2,3)}=\bar{r}_{1}, \\
& r_{\nu(1,1)}=r_{\nu(2,2)}=r_{\nu(2,4)}=r_{2}, \quad r_{\nu(1,3)}=r_{\nu(2,5)}=\bar{r}_{2}, \\
& \eta_{11}=\eta_{25}=x_{1} x_{2} \bar{x}_{1}, \quad \eta_{12}=\eta_{23}=x_{1}, \quad \eta_{13}=\eta_{22}=x_{2}, \\
& \eta_{14}=\left[x_{2}, x_{1}\right], \quad \eta_{15}=\eta_{21}=x_{2} x_{1} \bar{x}_{2}, \quad \eta_{24}=\left[x_{1}, x_{2}\right] .
\end{aligned}
$$


So applying the construction in Theorem 2.6 one obtains the following homology boundary link $L_{2}$, as shown in Figure 2.13 which admits $P$ as a pattern, via some epimorphism $\Phi: \pi_{1}\left(S^{3}-L\right) \rightarrow F$ where $\Phi\left(\mu_{i}\right)=x_{i}$ for $i=1,2$. Note that $L_{2}$ is the fusion of a 12-component trivial link along $10\left(=\sum \lambda_{i}\right)$ fusion bands [3], [4].

\section{Generalizations to $\mathbb{E}$-Links}

With some small modifications, we can extend Theorem 2.6 to a more general class of links, namely $\mathbb{E}$-links [3], [16].

Definition 3.1. A group $E$ is an $\mathbb{E}$-group if there exists a (not necessarily finite) 2-complex $K$ such that:

(i) $\pi_{1}(K)=E$,

(ii) $H_{1}(K)$ is torsion-free, and

(iii) $H_{2}(K)=0$.

If $K$ is a finite complex, then $E$ is said to be a finite $\mathbb{E}$-group.

All free groups are necessarily $\mathbb{E}$-groups. $\mathbb{E}$-groups are a more general class of groups than free groups. For example, any group $E$ whose deficiency is equal to $\operatorname{rank}\left(H_{1}(E)\right)$ is also an $\mathbb{E}$-group [3], [16].

Definition 3.2. Suppose $E$ is an $\mathbb{E}$-group with $\operatorname{rank}\left(H_{1}(E)\right)=m$. An E-calibration is a pair $\left(E,\left\{y_{1}, \ldots, y_{m}\right\}\right)$ where the $\left\{y_{1}, \ldots, y_{m}\right\}$ normally generates $E$.

An $E$-calibration is the analog of a pattern for $\mathbb{E}$-groups. Note than an $E$ calibration can only exist for $\mathbb{E}$-groups which are normally generated by $m$ elements where $m=\operatorname{rank}\left(H_{1}(E)\right)$.

Definition 3.3. A link $L$ is said to be an $\mathbb{E}$-link if it admits an $E$-calibration for some $\mathbb{E}$-group $E$.

In [3], it is shown that every sublink of a homology boundary link is an $\mathbb{E}$-link. With a small additional assumption (it is still unknown whether this assumption is necessary), Levine showed that every finite $\mathbb{E}$-link is a sublink of a homology boundary link [16], [18].

Given a finitely generated $\mathbb{E}$-group $E$ normally generated by elements $\left\{y_{1}, \ldots, y_{m}\right\}$, where $m=\operatorname{rank}\left(H_{1}(E)\right)$, one can construct a second $\mathbb{E}$-group $E^{\prime}$ of rank $m$ such that:

(i) there exists an epimorphism $\phi: E^{\prime} \rightarrow E$,

(ii) $E^{\prime}$ is normally generated by $y_{i}^{\prime}$ where $\phi\left(y_{i}^{\prime}\right)=y_{i}$, and

(iii) $E^{\prime}$ has the following presentation:

$$
E^{\prime}=\left\langle x_{1}^{\prime}, \ldots, x_{m^{\prime}}^{\prime}, y_{1}^{\prime}, \ldots, y_{m}^{\prime} \mid x_{i}^{\prime}=\prod_{j=1}^{\lambda_{i}} \eta_{i j} y_{\nu(i, j)}^{\prime \varepsilon_{i j}} \bar{\eta}_{i j}\right\rangle,
$$

where $\left\{x_{i}^{\prime}\right\}, 1 \leq i \leq m^{\prime}$, generate $E^{\prime}\left(m \leq m^{\prime}\right)$.

Construct $E^{\prime}$ as follows. Suppose $E$ is generated by $\left\{x_{1}^{\prime}, \ldots, x_{m^{\prime}}^{\prime}\right\}$. Then the free group on $\left\{x_{i}^{\prime}\right\} \cup\left\{y_{i}^{\prime}\right\}$ maps onto $E$ by $\phi\left(x_{i}^{\prime}\right)=x_{i}, \phi\left(y_{i}^{\prime}\right)=y_{i}$. Since $\left\{y_{i}\right\}$ normally generate $E$, this map factors through the group $E^{\prime}$ above. Consider a 2-complex $K^{\prime}$ consisting of the wedge product of $m$ 1-cells with $m^{\prime}$ 2-cells attached giving the presentation listed above. By construction, $K^{\prime}$ satisfies Definition 3.1, so $E^{\prime}$ is a finite $\mathbb{E}$-group. 
In order to generalize Theorem 2.6, we would like the collection $\left\{y_{i}\right\}$ to play the role that the pattern did in the case of homology boundary links. Note that it is sufficient to realize $\left(E^{\prime},\left\{y_{i}^{\prime}\right\}\right)$ as an $E^{\prime}$-calibration for $L$ since we can then map $\left(E^{\prime},\left\{y_{i}^{\prime}\right\}\right)$ onto $\left(E,\left\{y_{i}\right\}\right)$ via $\phi$ to obtain an $E$-calibration for $L$. The immediate obstruction is that the group $E^{\prime}$ (and $E$ ) onto which the calibration maps is not necessarily a free group and, worse yet, may not even be generated by the same number of elements as in the normal generating set $\left\{y_{i}\right\}$, i.e. $m^{\prime} \geq m$. This obstruction is overcome by beginning with a trivial link of more components and fusing more bands to obtain the desired $\mathbb{E}$-link $L$.

Theorem 3.4. Given any finitely generated $\mathbb{E}$-group $E$, calibration $\left(E,\left\{y_{1}, \ldots\right.\right.$, $\left.y_{m}\right\}$ ), and positive integer $n$, there exists an $\mathbb{E}$-link $L$ in $S^{n+2}$ admitting the $E$ calibration $\left(E,\left\{y_{1}, \ldots, y_{m}\right\}\right)$. In particular, $L$ is a ribbon link.

Proof. Let $\left\{y_{1}, \ldots, y_{m}\right\}$ be a normal generating set for $E$. By the remarks preceding the statement of the theorem, we may assume that $E$ has presentation:

$$
E=\left\langle x_{1}, \ldots, x_{m^{\prime}}, y_{1}, \ldots, y_{m} \mid x_{i}=\prod_{j=1}^{\lambda_{i}} \eta_{i j} y_{\nu(i, j)}^{\varepsilon_{i j}} \bar{\eta}_{i j}\right\rangle .
$$

Follow Theorem 2.6 and begin the construction by drawing $m$ circles one below another; orient all $m$ circles counterclockwise and call these circles $\beta_{k}$. Next to the $\beta_{k}$ 's draw $m^{\prime}$ sets of $\lambda_{i}$ concentric circles; call these circles $\alpha_{i j}$. Orient the circles $\beta_{k}$ and $\alpha_{i j}$ as in Theorem 2.6 for $1 \leq k \leq m, 1 \leq i \leq m^{\prime}$, and $1 \leq j \leq \lambda_{i}$. For simplicity, let $R_{i j}=\eta_{i j} y_{\nu(i, j)}^{\varepsilon_{i j}} \bar{\eta}_{i j}$; that is, let $R_{i j}$ be the $j$ th conjugate in the relation expressing $x_{i}$ as a product of conjugates in the presentation for $E$. Orient $\alpha_{i j}$ clockwise if the exponent $\varepsilon_{i j}$ of $y_{\nu(i, j)}$ in $R_{i j}$ is +1 and counterclockwise if -1 . Call the resulting link $L_{0}$ (see Figure 3.5).

Note that $L_{0}$ is a trivial link of $m+\sum_{i=1}^{m^{\prime}} \lambda_{i}$ components. Following the construction in Theorem 2.6, add $\sum_{i=1}^{m^{\prime}} \lambda_{i}$ fusion bands to $L_{0}$ which connect $\alpha_{i j}$ to $\beta_{\nu(i, j)}$ to obtain the desired $\mathbb{E}$-link $L$. One then proceeds exactly as in Theorem 2.6 to obtain an $E$-calibration $\Psi$ for $L$, completing the proof. $\diamond$

As was the case with the proof of Theorem 2.6, the proof of this theorem can be interpreted in the obvious way to produce $\mathbb{E}$-links of any dimension. Lastly, we close with an example illustrating the theorem. 

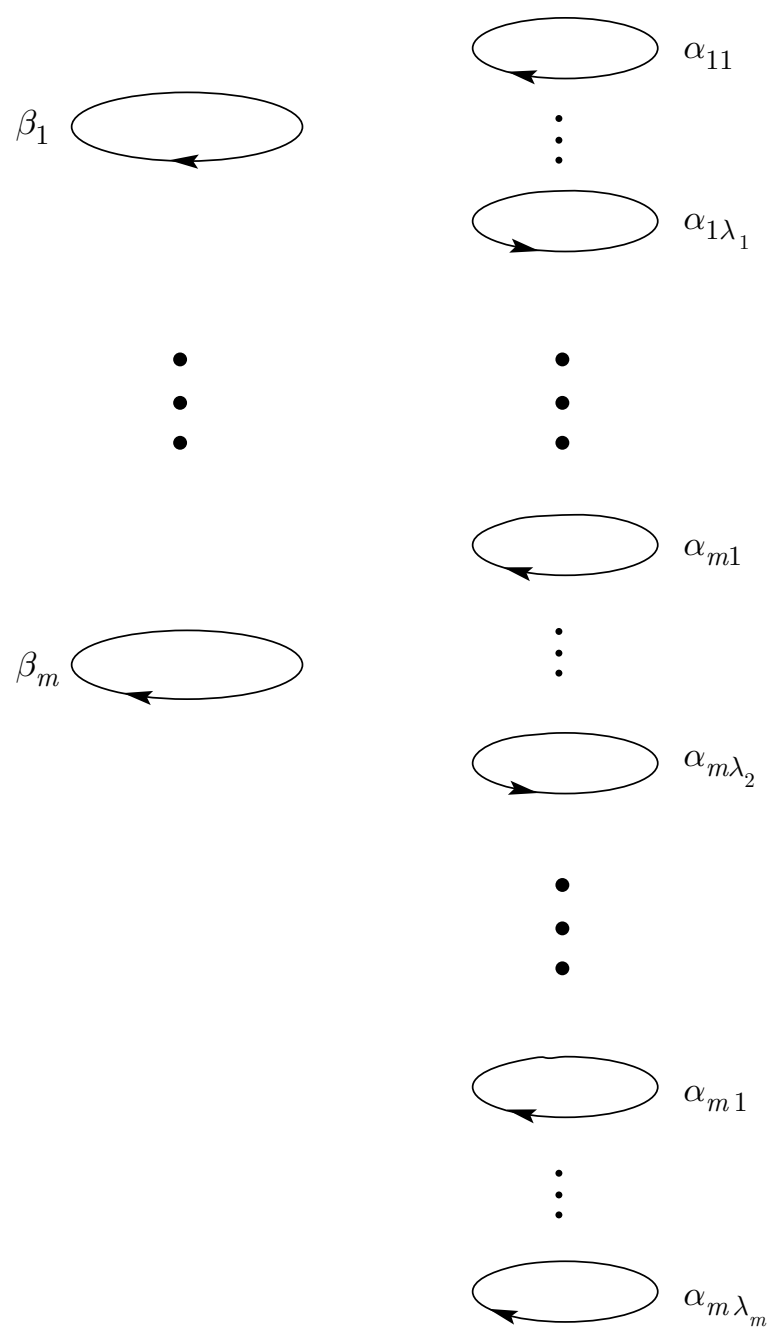

$L_{0}$

FiguRE 3.5

Example 3.6. Consider the $\mathbb{E}$-group with presentation

$$
E=\left\langle x_{1}, x_{2}, x_{3} \mid \bar{x}_{1}\left[x_{3}, x_{1}\right]\left[x_{3}, x_{2}\right]\right\rangle .
$$

Using $\left\{x_{2}, x_{3}\right\}$ as a normal generating set for $E$, we may equivalently present $E$ by

$$
\left\langle x_{1}, x_{2}, x_{3}, y_{1}, y_{2} \mid x_{1}=y_{2} x_{1} \bar{y}_{2} \bar{x}_{1} x_{3} y_{1} \bar{x}_{3} \bar{y}_{1}, x_{2}=y_{1}, x_{3}=y_{2}\right\rangle .
$$

G. Baumslaug [1] has shown that $E$ is parafree but not free, and therefore cannot map onto a free group of rank 2 . Hence, any $\mathbb{E}$-link $L$ calibrating $E$ cannot be a homology boundary link; however $L$ will be a sublink of a homology boundary link [10]. Following the construction outlined in Theorem 3.4, a link $L$ calibrating $E$ is shown in Figure 3.7. Observe that the meridians $\mu_{1}$ and $\mu_{2}$ labeled in the diagram 


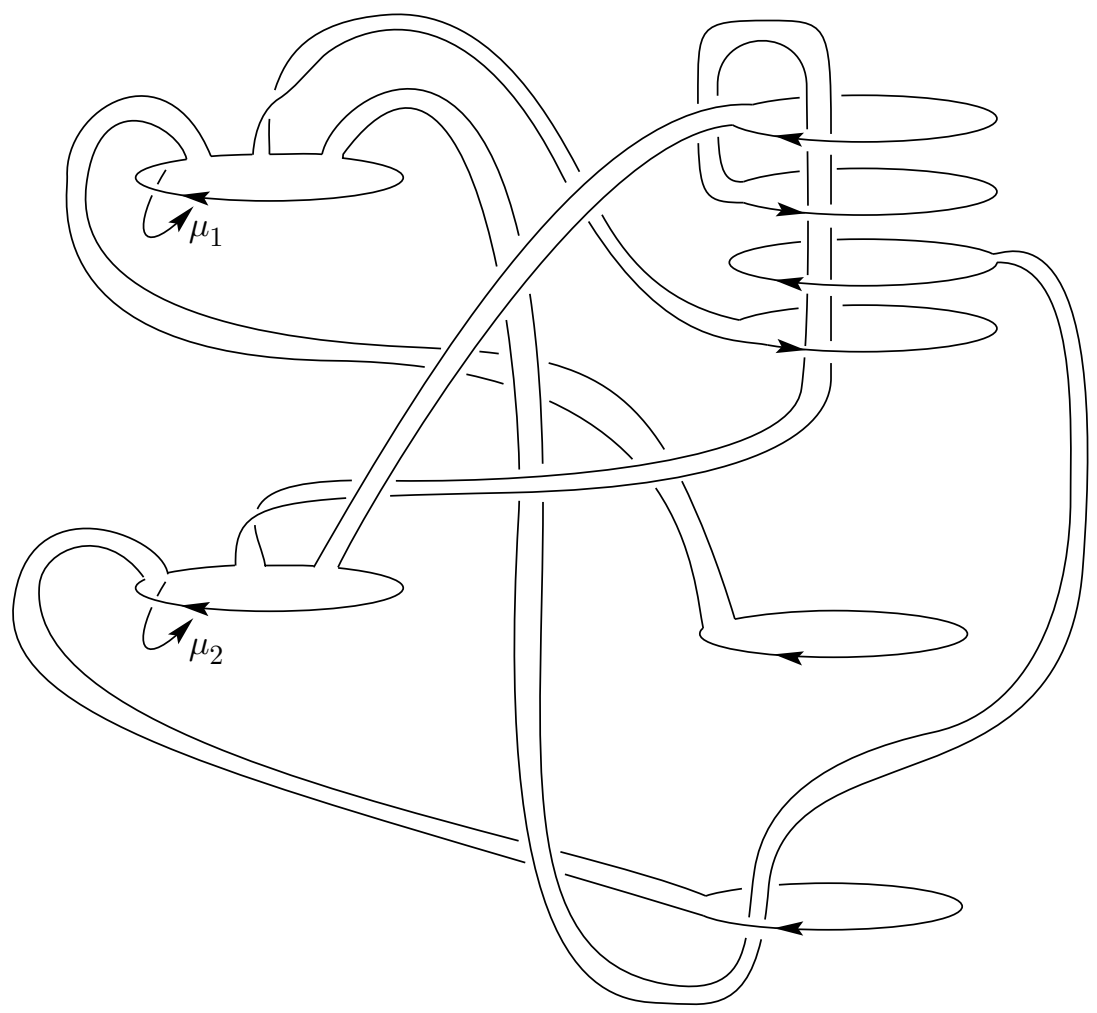

$L$

FIGURE 3.7

are the meridians which the $E$-calibration will map to the normal generating set $\left\{y_{1}=x_{2}, y_{2}=x_{3}\right\}$ in $E$.

$$
\begin{aligned}
& \lambda_{1}=4, \quad \lambda_{2}=\lambda_{3}=1, \\
& r_{\nu(1,1)}=y_{2}, \quad r_{\nu(1,2)}=\bar{y}_{2}, \quad r_{\nu(1,3)}=y_{1}, \quad r_{\nu(1,4)}=\bar{y}_{1}, \\
& r_{\nu(2,1)}=y_{1}, \quad r_{\nu(3,1)}=y_{2}, \\
& \eta_{12}=x_{1}, \quad \eta_{13}=x_{3}, \quad \eta_{11}=\eta_{14}=\eta_{21}=\eta_{31}=1 .
\end{aligned}
$$

\section{REFERENCES}

[1] G. Bausmlaug, Groups with the Same Lower Central Sequence as a Relatively Free Group, Trans. Amer. Math. Soc. 142 (1969), 507-38. MR 39:6959

[2] S. Cappell and J. Shaneson, Link Cobordism, Comment. Math. Helv. 55 (1980), 20-49. MR 81j:57011

[3] T. Cochran, Link Concordance Invariants and Homotopy Theory, Invent. Math. 90 (1987), 635-45. MR 89f:57033

[4] _ Derivatives of Links: Milnor's Concordance Invariants and Massey's Products, \#427, Memoirs of the Amer. Math. Soc. 84 (1990), Providence, RI. MR 91c:57005

[5] T. Cochran and J. Levine, Homology Boundary Links and the Andrews-Curtis Conjecture, Topology 30 (1991), 231-9. MR 92f:57011 
[6] T. Cochran and K. Orr, Not All Links are Concordant to Boundary Links, Bull. Amer. Math. Soc. (N.S.) 23 (1990), 99-106. MR 91c:57012

[7] _ Not All Links are Concordant to Boundary Links, Ann. of Math. 138 (1993), 519-54. MR 95c:57042

[8] - Homology Boundary Links and Blanchfield Forms: Concordance Classification and New Tangle-Theoretic Constructions, Topology 33 (1994), 397-427. MR 95f:57041

[9] R. De Meo, Cobordisms of Non-boundary Links, Ph.D. Dissertation, Princeton University, 1980.

[10] J. Hillman, Alexander Ideals of Links, Springer-Verlag Lecture Notes in Math. 895, Springer, Berlin, 1981. MR 84j:57004

[11] U. Kaiser, Homology Boundary Links and Fusion Constructions, Osaka J. Math. 29 (1992), 573-93. MR 93h:57038

[12] M. Kervaire, Les noeuds de dimensions supérieures, Bull. Soc. Math. France 93 (1965), 225-71. MR 32:6479

[13] K. Ko, Seifert Matrices and Boundary Link Cobordisms, Trans. Amer. Math. Soc. 299 (1987), 657-81. MR 88h:57018

[14] J. Levine, Knot Cobordism Groups in Codimension Two, Comment. Math. Helv. 44 (1969), 229-44. MR 39:7618

[15] _ Link Concordance and Algebraic Closure of Groups, Comment. Math. Helv. 64 (1989), 236-55. MR 91a:57016

[16] _ Link Concordance and Algebraic Closure II, Invent. Math. 96 (1989), 571-92. MR 91g:57007

[17] _ Link Invariants Via the Eta Invariant, Comment. Math. Helv. 69 (1994), 82-119. MR 95a:57009

[18] J. Levine, W. Mio, and K. Orr, Links With Vanishing Homotopy Invariants, Comm. Pure Appl. Math. 46 (1993), 213-20. MR 94e:57036

[19] W. Mio, On Boundary Link Cobordism, Math. Proc. Cambridge Philos. Soc. 101 (1987), 259-66. MR 88e:57023

[20] N. Smythe, Boundary Links, Topology Seminar: Wisconsin, 1965 (ed. R. H. Bing), Annals of Math. Studies 60, Princeton Univ. Press, Princeton, NJ, pp. 59-72.

Department of Mathematics, Rice University, P. O. Box 1892, Houston, Texas 772511892

Current address: 7932 Butterfield Dr., Elkridge, Maryland 21075

E-mail address: apbellis@erols.com 